\title{
Controlled placement and electrical contact properties of individual multiwalled carbon nanotubes on patterned silicon chips
}

\author{
Y. F. Hsiou ${ }^{\mathrm{a})}$ and Y. J. Yang \\ Graduate Institute of Electronics Engineering and Department of Electrical Engineering, National Taiwan \\ University, Taipei, Taiwan, Republic of China \\ L. Stobinski \\ Institute of Physical Chemistry, Polish Academy of Sciences, 01-224 Warsaw, Kasprzaka 44/52, Poland \\ Watson Kuo ${ }^{\mathrm{a})}$ \\ Department of Physics, National Chung Hsing University, Taichung, Taiwan, Republic of China \\ C. D. Chen ${ }^{\text {b) }}$ \\ Institute of Physics, Academia Sinica, Taipei, Nankang 11529, Taiwan, Republic of China
}

(Received 2 July 2003; accepted 9 December 2003)

\begin{abstract}
A scheme that allows on-chip growth of multiwalled carbon nanotubes at designed locations is demonstrated. The nanotubes were grown by thermal chemical vapor deposition and were contacted to nanoscaled $\mathrm{Cr}$ electrodes fabricated by standard e-beam lithography techniques. The contacts were found to be Ohmic with resistance values on the order of $10^{3} \Omega$ at room temperature. Remarkably, the contacts showed weak temperature dependence down to $40 \mathrm{mK}$ and were insensitive to the magnetic field up to 5 T. (C) 2004 American Institute of Physics.
\end{abstract}

[DOI: $10.1063 / 1.1645985]$

The transport phenomena in carbon nanotubes (CNTs) have attracted much attention in recent years due to their unique electronic band structures ${ }^{1}$ and their potential applications as nanoscaled electronic devices. ${ }^{2}$ In this field, one of the major concerns is the electrical contact between the CNTs and the electrodes. Presently, there are two approaches for making electrical contact. In the first approach, CNTs are dispersed onto chips with prefabricated electrodes. ${ }^{3}$ However, since for many practical applications only one CNT across the electrodes is desired, this approach requires not only knowledge about the CNT-substrate interface properties, but also experiences in the manipulation of CNTs. In the second approach, CNTs are first randomly dispersed over the substrates and the electrodes are then placed on the top of the selected CNTs. ${ }^{2}$ This approach often suffers from poor controllability of the locations of the CNTs, and it further requires additional efforts to search for the randomly spread CNTs. Moreover, the contact between the electrodes and the nanotubes usually presents a serious problem. The measured resistance reported in the literature ranges from $10^{3}$ to $10^{6} \Omega$ at room temperature, ${ }^{3,4}$ and has somewhat of a dependence on the electrode materials. ${ }^{5,6}$ Attempts made on the contact issues have raised some possible schemes (with unclear origins), including annealing, ${ }^{7}$ electron irradiation, ${ }^{3}$ and use of early $3 \mathrm{~d}$ elements as electrode materials. ${ }^{6}$ In this letter, we propose and demonstrate a scheme that allows on-chip growth of the CNTs and control of the CNT locations, and provides good electrical contacts. This method utilized thermal chemical vapor deposition for synthesis of multiwalled carbon nanotubes (MWNTs) and electron-beam lithography for preparation of nanoscaled electrodes. When using $\mathrm{Cr}$ as contact electrode material, we found that the contact resis-

a) Also at Institute of Physics, Academia Sinica, Taipei, Nankang 11529, Taiwan, Republic of China.

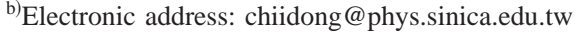

tance was on the order of $10^{3} \Omega$. The low contact resistance possessed weak temperature and magnetic-field dependences, which is desirable for many applications.

The fabrication process is described as follows. First, large contact pads and leads consisting of a 40-nm-thick Pt layer on top of a 10-nm-thick Ti layer were prepared by standard photolithography and lift-off techniques. The substrates were Si wafers coated with a 300-nm-thick thermally grown $\mathrm{SiO}_{2}$ layer. Using the fabricated leads as fiducial marks, 9-nm-thick thermally evaporated iron squares $9 \mu \mathrm{m}^{2}$ in area were made at the designed locations by e-beam lithography and lift-off techniques. In the MWNT growth process, the chips were heated to $\sim 700^{\circ} \mathrm{C}$ and the native oxidation layer on the surface of iron squares was reduced to iron in a $1 \mathrm{mBar}$ hydrogen ambiance for $5 \mathrm{~min}$. After this treatment, the surface of the iron squares became very rough, and nanoscaled iron particles were formed on the roughened squares. These iron nanoparticles then served as a catalyst for thermal chemical vapor deposition growth of MWNTs. After the chip was cooled down to room temperature, the hydrogen gas was then pumped out, pure ethylene was subsequently introduced into the chamber to a pressure of 1 $\mathrm{mBar}$, and the chip was heated to $\sim 750{ }^{\circ} \mathrm{C}$ for $5 \mathrm{~min}$. After this step, straight MWNTs with lengths of about a few microns were produced. The length of the tube is presumably determined by the elevation angle of the precipitation at the initial growth stage. As this angle may change with the strength of interaction between the catalytic nanoparticles and the underneath substance, thick iron films, which tend towards a smaller elevation angle, were futile for the growth of long $(\sim 10 \mu \mathrm{m})$ MWNTs.

Individual MWNTs with lengths of 7-10 $\mu \mathrm{m}$ were sticking out of the iron squares and lying on the $\mathrm{SiO}_{2}$ surface. The outer diameters of these tubes ranged from 10 to 50 


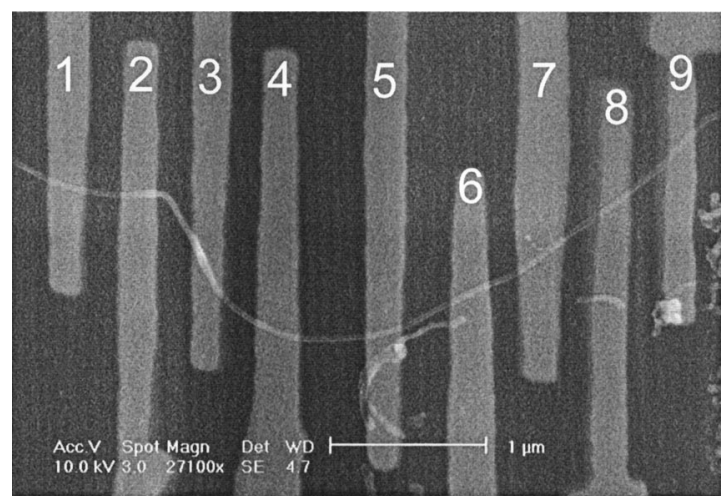

FIG. 1. An SEM image of one of the measured MWNTs. The tube was grown from an iron catalyst (not shown in this image) and was connected to nine $\mathrm{Cr}$ electrodes.

$\mathrm{nm}$. Tests were performed to ensure that the MWNTs were firmly attached to the substrate and could withstand the mechanical forces in subsequent lithographic processes. Finally, the positions of the selected MWNTs were carefully determined by the scanning electron microscope (SEM) images and were then used for designing the electrical leads. Figure 1 shows an SEM image of a fabricated sample. The electrodes were arranged in a multiprobe configuration and were placed on the top of the selected MWNTs by means of e-beam lithography and lift-off techniques. These electrodes were $200 \mathrm{~nm}$ wide and the edge-to-edge spacing between the electrodes was about $300 \mathrm{~nm}$. They were made of 30-nmthick $\mathrm{Cr}$ and were covered by a 10 -nm-thick protective $\mathrm{Au}$ layer. In addition to the measurement electrodes, a side-gate electrode, about $500 \mathrm{~nm}$ away from the tube, was made in the same lithographic step.

Current-voltage $(I-V)$ characteristics were measured using two-probe and four-probe techniques. Figure 2(a) shows two-probe $I-V$ characteristics for one of the MWNTs measured at several temperatures. The room-temperature $I-V$ characteristics were linear, suggesting an Ohmic contact or a linear tunnel junction between the leads and the MWNTs. Four-probe $I-V$ characteristics are depicted in Fig. 2(b). Both the tube resistance $R_{4 t}$ between pairs of neighboring electrodes measured using the standard four-probe technique and the resistance between pairs of two neighboring electrodes, $R_{2 t}$, were in the range from $10^{3}$ to $10^{4} \Omega$. Only for a few tubes was the resistance higher, and was approximately $10^{4}-10^{5} \Omega$. The measured $R_{2 t}$ and $R_{4 t}$ for the sample shown in Fig. 1 are listed in Table I. Although the resistances of each individual contact cannot be determined, the values of the contact resistance are no greater than $\left(R_{2 t}-R_{4 t}\right)$, which is on the order of $10^{3} \Omega$. At temperatures below $4 \mathrm{~K}$, two-probe $I$ - $V$ characteristics generally showed a slight nonlinear behavior with current smaller than the linear ones. Interestingly, four-probe measurements also revealed very similar $I-V$ characteristics. Furthermore, the current at low bias voltages showed periodic modulation upon application of gate voltages. This can probably be attributed to intratube Coulomb blockade tunneling and is a subject for further studies.

A multiprobe technique can provide a more precise estimate of the contact resistance. In this technique, it is assumed that the length of the contact region is much smaller than the length of the tube between the two neighboring Downloaded 09 Mar 2009 to 140.112.113.225. Redistribution subject
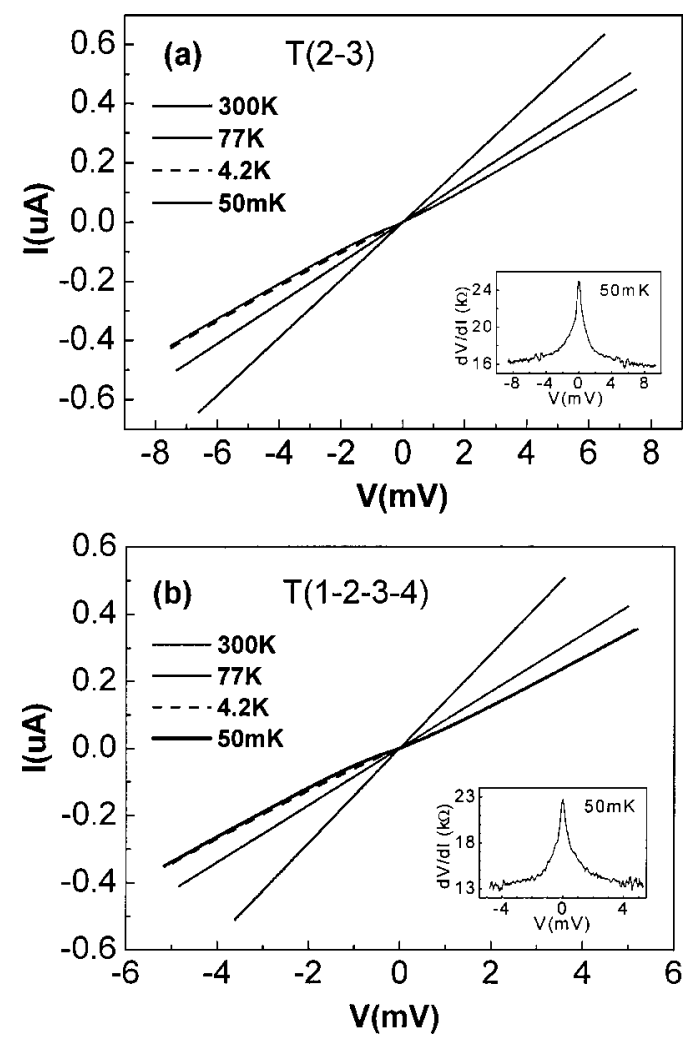

FIG. 2. $I-V$ characteristics at $300 \mathrm{~K}, 77 \mathrm{~K}, 4 \mathrm{~K}$, and $50 \mathrm{mK}$, measured by (a) two-probe and (b) four-probe techniques. The resistance increases with decreasing temperature. The $I-V$ characteristics at $4 \mathrm{~K}$ and $50 \mathrm{mK}$ are almost identical, and show nonlinear behavior around zero bias voltage. Insets in (a) and (b) show $d V / d I$ vs $V$ at $50 \mathrm{mK}$.

contacts. In an $m$-probe configuration, one can estimate contact resistances $R_{C n}$ of the $n$th probe and the tube resistance $R_{T n, n+1}$ between a pair of neighboring probes $(n, n+1)$ by using the following relations:

$$
\begin{aligned}
R_{C n}= & \left(R_{n-1, n}+R_{n, n+1}-R_{n-1, n+1}\right) / 2 \\
& \text { for } n=2 \text { to } m-1, \\
R_{T n, n+1}= & \left(R_{n-1, n+1}+R_{n, n+2}-R_{n-1, n}-R_{n+1, n+2}\right) / 2 \\
& \text { for } n=2 \text { to } m-2,
\end{aligned}
$$

where $R_{i, j}$ denotes the measured resistance between probes $i$ and $j$, and $n=1$ denotes the first probe at one of the two ends. Using this technique, the room-temperature resistances of the sample displayed in Fig. 1 were calculated, and the results are listed in Table I. Here, we should note that, as the width of the electrodes is not small compared with the length of the tube between two neighboring electrodes (see Fig. 1), the conditions for this formula are not strictly fulfilled. Nev-

TABLE I. Room-temperature two-probe and four-probe resistances, $R_{i j}$ and $R_{4 t}$, and calculated values of contact resistance $R_{C j}$ and tube resistance $R_{T i j}$ for tube $T(h, i, j, k)$. Note the very close values between the measured and the calculated tube resistances.

\begin{tabular}{cccccc}
\hline \hline$T(h-i-j-k)$ & $R_{2 t} \equiv R_{i, j}(\mathrm{k} \Omega)$ & $R_{4 t}(\mathrm{k} \Omega)$ & $R_{C i}(\mathrm{k} \Omega)$ & $R_{C j}(\mathrm{k} \Omega)$ & $R_{T, i, j}(\mathrm{k} \Omega)$ \\
\hline$T(1-2-3-4)$ & 10.24 & 7.09 & 1.46 & 1.68 & 7.10 \\
$T(2-3-4-5)$ & 7.74 & 4.10 & 1.68 & 1.96 & 4.10 \\
$T(4-5-7-8)$ & 13.57 & 8.11 & 2.28 & 3.18 & 8.11 \\
$T(5-7-8-9)$ & 8.55 & 2.74 & 3.18 & 2.63 & 2.74 \\
$T(3-4-5-7)$ & 10.92 & 6.70 & 1.96 & 2.28 & 6.68 \\
\hline \hline
\end{tabular}




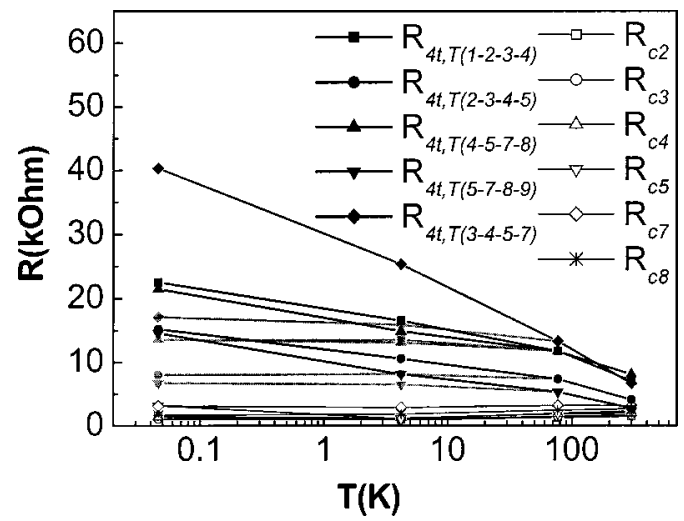

FIG. 3. The tube resistance $\left(R_{4 t, T(h, i, j, k)}\right)$ and contact resistance $\left(R_{c j}\right)$ at 300 $\mathrm{K}, 77 \mathrm{~K}, 4 \mathrm{~K}$, and $50 \mathrm{mK}$. For all tubes, the tube resistances increase with decreasing temperature, and, as depicted in Fig. 2(b), this increase is accompanied with nonlinear $I-V$ characteristics at low bias voltage. The dark curves correspond to zero-bias resistances and the gray curves correspond to asymptote resistances.

ertheless, the tube resistances obtained in this way were found to be very close to the values of the measured $R_{4 t}$, indicating the validity of this multiprobe technique. This validity can be explained by noting that the voltage probes give the values of the average tube voltage within the contact region, provided that the contacts are uniform over the region. Applying Eq. (1) on many MWNTs, we conclude that the room-temperature contact resistances are mostly on the order of $10^{3} \Omega$, but the tube resistances may vary between $10^{3}$ and $10^{5} \Omega / \mu \mathrm{m}$. Figure 3 shows the temperature dependence of the tube resistances and contact resistances. The tube resistance may increase very much upon cooling. Comparing the estimated contact resistances at various temperatures, we found that the contact resistance showed no sensitive temperature dependence even at temperatures down to $40 \mathrm{mK}$, suggesting that $\mathrm{Cr}$ is a good contact metal for MWNTs. This low Ohmic contact resistance may be attributed to the closeness of the work functions of $\mathrm{Cr}$ and carbon nanotubes. The reported values of carbon nanotubes range between 4.5 and $4.8 \mathrm{eV},{ }^{8}$ fairly close to the reported value of $4.4-4.5 \mathrm{eV}$ for $\mathrm{Cr}$. This would provide a much better Ohmic metal-tube contact than other frequently used electrode materials, such as $\mathrm{Au}(\sim 5.1 \mathrm{eV})$ or $\mathrm{Pt}(\sim 5.7 \mathrm{eV})$.

Magnetic-field dependence of the $\mathrm{Cr}$ contacts was also studied. Figure 4 shows the asymptote resistances measured by both two-probe and four-probe techniques in perpendicularly applied magnetic fields up to $5 \mathrm{~T}$. These curves indicate that even the tube resistances decrease with the applied fields; the contact resistances are essentially unchanged. The gradual decreasing of the tube magnetoresistance is suggestive of reduction of the band gap. ${ }^{9}$ It is interesting to note that, as depicted in the inset of Fig. 4, some other tubes (not listed in Table I) exhibited a negative magnetoresistance with a rapid decrease at low fields, which is a sign of weak localization behavior. In additional to that, plateau structures appeared at fields around 2.2 and $4.5 \mathrm{~T}$. This could probably be attributed to Aharonov-Bohm effects on a trajectory containing a magnetic flux quantum. ${ }^{10}$ As an estimate, the projected area normal to a field period of $2.2 \mathrm{~T}$ is about $1000 \mathrm{~nm}^{2}$, which is a fraction of the cross-section area of $4500 \mathrm{~nm}^{2}$ (electrode separation $\approx 150 \mathrm{~nm}$, tube diameter $\approx 30 \mathrm{~nm}$ ). This

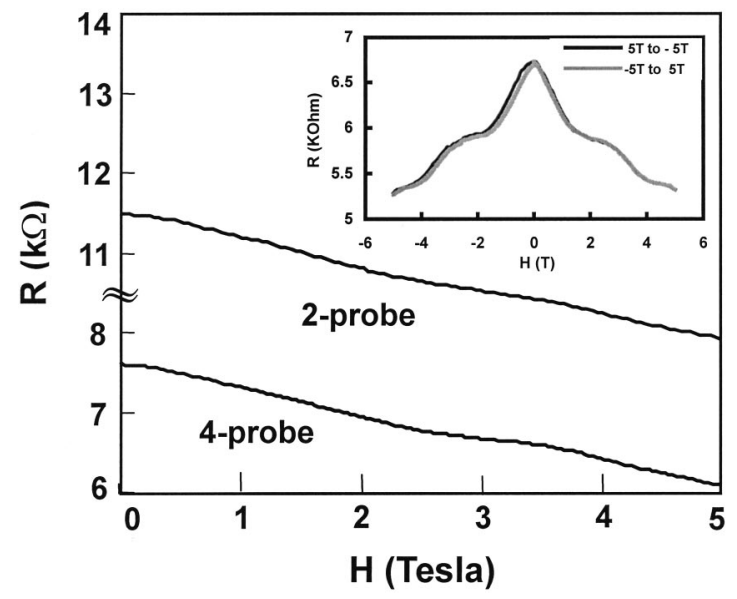

FIG. 4. The asymptote resistance at $70 \mathrm{mK}$ as a function of the perpendicularly applied field. Both two-probe and four-probe magnetoresistance decreases with increasing field. A comparison between the two curves indicated that the contact resistances are unaffected by the applied fields. The inset shows four-probe magnetoresistance of a separate tube.

trajectory is sample-specific and can vary from tube to tube. However, it strongly suggests that the phase-breaking length of the tube is greater than $150 \mathrm{~nm}$. On the other hand, the low resistance of the tube $\left(R_{4 t}<R_{Q} / 2\right.$, where $R_{Q}=13.6 \mathrm{k} \Omega$ is the quantum resistance) suggests that the transport is contributed by more than one shell of the MWNTs.

We have also tested other electrode materials. The contact resistances with $\mathrm{Ti} / \mathrm{Au}$ electrodes were about $10^{6}-10^{7} \Omega$, which were much higher than the reported values. ${ }^{11}$ Aluminum electrodes were tested on more than 10 samples, but the contact resistances were found to be very high, usually larger than $10^{9} \Omega$, contradicting the theoretical prediction. $^{5}$

In conclusion, we have demonstrated a reliable method for synthesis and controlled placement of MWNTs. We also conclude that $\mathrm{Cr}$ is a good candidate for contact material. It provides low Ohmic contact resistance with weak temperature and magnetic-field dependences.

This research is funded by the National Science Council, Grant Nos. 89-2112-M-001-033 and 91-2120-M-001-001.

${ }^{1}$ R. Saito, G. Dresselhaus, and M. S. Dresselhaus, Physical Properties of Carbon Nanotubes (Imperial College Press, London, 1998).

${ }^{2}$ A. Bachtold, P. Hadley, T. Nakanishi, and C. Dekker, Science 294, 1317 (2001).

${ }^{3}$ A. Bachtold, M. Henny, C. Terrier, C. Strunk, C. Schönenberger, J.-P. Salvetat, J.-M. Bonard, and L. Forró, Appl. Phys. Lett. 73, 274 (1998).

${ }^{4}$ S. Frank, P. Poncharal, Z. L. Wang, and W. A. de Heer, Science 280, 1744 (1998).

${ }^{5}$ J. Teresoff, Appl. Phys. Lett. 74, 2122 (1999).

${ }^{6}$ A. N. Andriotis, M. Menon, and G. E. Froudakis, Appl. Phys. Lett. 76, 3890 (2000).

${ }^{7}$ Y. Zhang, T. Ichihashi, E. Landree, F. Nihey, and S. Iijima, Science 285, 1719 (1999).

${ }^{8}$ S. J. Tans, A. R. M. Verschueren, and C. Dekker, Nature (London) 393, 49 (1998); R. Gao, Z. Pan, and Z. L. Wang, Appl. Phys. Lett. 78, 1757 (2001).

${ }^{9}$ L. Langer, V. Bayot, E. Grivei, J.-P. Issi, J. P. Heremans, C. H. Olk, L. Stockman, C. Van Haesendonck, and Y. Bruynseraede, Phys. Rev. Lett. 76, 479 (1996).

${ }^{10}$ A. Bachtold, C. Strunk, J.-P. Salvetat, J.-M. Bonard, L. Forró, T. Nussbaumer, and C. Schönenberger, Nature (London) 397, 673 (1999).

${ }^{11}$ Z. Yao, C. L. Kane, and C. Dekker, Phys. Rev. Lett. 84, 2941 (2000). 